\title{
Medical Image of the Week: Portal Vein Thrombosis in a Patient with Polycythemia Vera
}

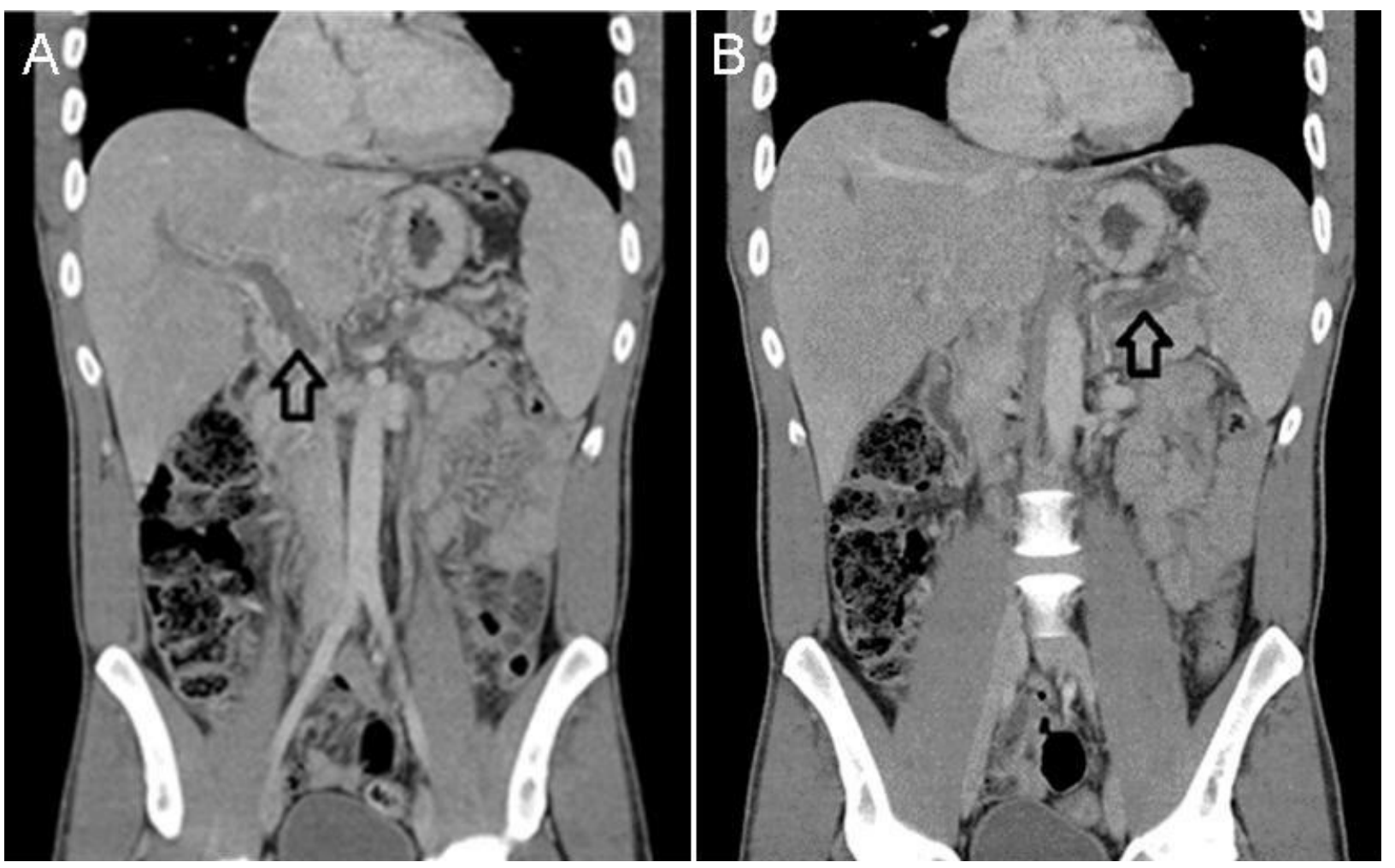

Figure 1 Panel A: CT of the abdomen demonstrating a thrombus within the portal vein (black arrow). Panel B: CT showing extension of the thrombus into the splenic vein (black arrow).

A 39-year-old man with no past medical history presented with acrocyanosis of his left second toe and right upper abdominal pain. Initial labs showed polycythemia, leukocytosis and thrombocytosis that were unchanged with intravenous fluid administration. A CT of the abdomen was obtained and showed portal vein thrombosis without evidence of cirrhosis (Figure 1). Subsequent studies revealed a positive JAK2 V617F mutation and low erythropoietin levels consistent with polycythemia vera (PV). The patient was placed on anticoagulation, low dose aspirin, and received phlebotomy.

PV is a chronic myeloproliferative neoplasm defined by an increase in red blood cell mass in the absence of a physiologic stimulus. The 2016 World Health Organization classification of myeloid neoplasms and acute leukemia has three major criteria and one minor criterion in the diagnosis of PV. Diagnosis requires either 3 major criteria or 2 major with 1 minor criterion (1).

Major Criteria for Polycythemia Vera

1. Increased hemoglobin level(>16.5 in men, $>16$ in women) or hematocrit( $>49$ in men, $>48$ in women)

2. Bone marrow biopsy showing hypercellularity for age with trilineage growth 


\section{JAK2 V617F or JAK2 exon 12 mutation}

Minor Criterion for Polycythemia Vera

1. Serum erythropoietin level below the reference range of normal.

Due to the propensity of unusual thrombosis once a diagnosis is made therapy should be initiated without delay. PV should be treated with phlebotomy followed by maintenance therapy with continued phlebotomy or hydroxyurea to maintain target hematocrit levels and with low dose aspirin $(2,3)$.

Hamayon Babary, MD and Muthena Maklad, MD Department of Internal Medicine University of Nevada School of Medicine: Las Vegas Las Vegas, NV USA

\section{References}

1. Arber DA, Orazi A, Hasserjian R, Thiele J, Borowitz MJ, Le Beau MM, Bloomfield CD, Cazzola M, Vardiman JW. The 2016 revision to the World Health Organization classification of myeloid neoplasms and acute leukemia. Blood. 2016 May 19;127(20):2391-405. [CrossRef] [PubMed]

2. Marchioli R, Finazzi F, Specchia G, et al. Cardiovascular events and intensity of treatment in polycythemia vera. N Engl J Med. 2013 Jan 3;368(1):22-33. [CrossRef] [PubMed]

3. Landolfi R, Marchioli R, Kutti J, Gisslinger H, Tognoni G, Patrono C, Barbui T; European Collaboration on Low-Dose Aspirin in Polycythemia Vera Investigators. Efficacy and safety of low-dose aspirin in polycythemia vera. N Engl J Med. 2004 Jan 8;350(2):114-24. [CrossRef] [PubMed] 